\title{
Risk of ulcer bleeding in patients infected with Helicobacter pylori taking non-steroidal anti-inflammatory drugs
}

Aalykke C, Lauritsen JM, Hallas J, et al. Helicobacter pylori and risk of ulcer bleeding among users of nonsteroidal anti-inflammatory drugs: a case-control study. Gastroenterology 1999;116:1305-9.

\section{Objective}

To determine whether Helicobacter pylori is an independent risk factor for bleeding peptic ulcer in users of nonsteroidal anti-inflammatory drugs (NSAIDs), including aspirin.

\section{Design}

A prospective matched case-control study.

\section{Setting}

Odense University Hospital, Denmark.

\section{Subjects}

132 patients with a bleeding peptic ulcer $(n=124)$ or haemorrhagic gastritis $(n=8)$ at endoscopy who had taken an NSAID in the previous week and 136 controls who had taken NSAIDs without gastrointestinal complications. The controls were recruited from rheumatology and geriatric outpatient clinics.

\section{Measurements}

$H$ pylori status assessed by serology and ${ }^{13} \mathrm{C}$-urea breath test and regarded as positive if either test was positive. Data on potential confounding factors including smoking and alcohol were collected by interview.

\section{Main result}

$H$ pylori was present in $57 \%$ of cases and $43 \%$ of controls. The adjusted odds ratio of bleeding from a peptic ulcer owing to $H$ pylori infection in NSAID users was 1.81 (95\% CI 1.02 to 3.21 ) and was similar in aspirin and non-aspirin NSAID users. Peptic ulcer bleeding was also statistically significantly associated with a history of previous ulcer bleeding, dyspepsia within the previous 3 months, drinking alcohol but not with smoking. About $16 \%$ of bleeding peptic ulcers in NSAID users could be attributed to $H$ pylori infection.

\section{Conclusion}

NSAID users infected with $H$ pylori have an almost doubled risk of bleeding peptic ulcer compared with uninfected NSAID users.

\section{Comment}

Helicobacter pylori and non-steroidal anti-inflammatory drugs (NSAIDs) between them cause the vast majority of peptic ulcers and their complications. However, their interaction remains extremely controversial. Aalykke et al's study aimed to address this issue by investigating $H$ pylori status in a group of patients presenting with bleeding peptic ulcer and matched controls, all of whom were taking NSAIDs. The study found a relatively small increase (odds ratio 1.81 ) associated with $H$ pylori infection, which only accounted for $16 \%$ of bleeding peptic ulcers in NSAID users. Although not investigated in this study, $H$ pylori accounts for nearly all bleeding peptic ulcers in nonNSAID users.

These data are closely in line with a meta-analysis of studies of NSAID users investigated by endoscopy which also found a 1.8-fold increase in risk of peptic ulcer ${ }^{1}$ in NSAID users infected with $H$ pylori compared with those not infected, although results from individual studies are variable. Unfortunately, there is also at least as much discrepancy in epidemiological studies of ulcer bleeding. Of six other studies, three have found no effect of $\mathrm{H}_{\text {pylori }}{ }^{2-4}$ and three have found evidence of possible protection, ${ }^{5-7}$ at least in some subgroups. The strength of the study by Aalykke et al compared with these other studies is that it involved a direct comparison restricted to NSAID using cases and controls. The study also has a number of weaknesses. Despite attempts to match and allow for the effect of age, it is unfortunate that controls were on average six years younger than cases. The study did not match for social class, and use of controls from the rheumatology clinic could have spuriously biased the study results if these controls were of a higher socioeconomic group.

The interaction between aspirin and non-aspirin NSAIDs and $H$ pylori may not be the same. Strictly speaking the study found no significant effect of $H$ pylori in aspirin and non-aspirin users investigated separately and required amalgamation for significance to be reached. In contrast to some other studies, the effect on gastric though not duodenal ulcer was statistically significant, although the latter could well be a type 2 error.

Taken at face value, Aalykke et al's study would suggest that $H$ pylori eradication would reduce ulcer disease in NSAID users by about $16 \%$. Again, direct data from eradication trials are somewhat discordant and controversial. One study found $H$ pylori eradication reduced the development of gastric ulcer much more than this at two months (from $26 \%$ to $7 \%$ ). ${ }^{8}$ It is difficult to explain why the reduction is so much bigger than the largest effect suggested by the most positive of the seven published epidemiological studies. One possibility is that this short term therapeutic study used a cytoprotective bismuth compound, leading to a confounded result. In a longer term study of $H$ pylori eradication using a non-bismuth based regimen, no effect of $H$ pylori eradication was seen. ${ }^{9}$ A third smaller study also showed no significant effect. ${ }^{10}$

Very different patients were investigated in these studies. The positive outcome involved patients essentially starting NSAIDs for the first time with no past history of dyspepsia or ulceration. This was the inverse of the group in which no effect was seen where pre-existing NSAID use, and endoscopically proved peptic ulcer and/or moderate to severe 
dyspepsia were the entry requirements. Likewise in studies relating outcome to natural status, healing rates were lower and relapse rates higher in $H$ pylori negative than positive individuals. ${ }^{11}$ Acid suppression is known to be more effective in $H$ pylori positive patients. ${ }^{12}$ However, even in patients not taking anti-ulcer treatment, studies such as that of Aalykke et al suggest the harmful effect of $H$ pylori is much less in NSAID users than in non-users, perhaps because toxicity is in whole or part abrogated by intrinsically beneficial actions such as an ability to blunt the reduction in gastric mucosal prostaglandin synthesis by NSAIDs.

Thus the relation between $H$ pylori and NSAIDs may vary with patient group. Eradication before ulceration has been induced could be protective but Aalykke et al's study suggests that the long term effect would be much smaller than was seen in the Hong Kong study and more data are needed before widespread $H$ pylori eradication in such patients can be recommended. It is equally clear that $H$ pylori eradication alone is an ineffective and unjustified approach in NSAID users that have had a previous peptic ulcer. These patients require anti-ulcer prophylaxis if they are to continue taking NSAIDs. If this prophylaxis involves acid suppression, its effectiveness will be reduced by $H$ pylori eradication. ${ }^{12}$ Whether remaining discrepancies can be explained by differences in the prokaryotic or eukaryotic populations of the different countries in which they have been carried out will be well worth investigating. However, it is likely that the impetus to do this will decline with increasing use of COX-2 inhibitors as these drugs appear neither to cause ulcers nor to interact significantly with $H$ pylori.
C J HAWKEY

Division of Gastroenterology,

University Hospital, Nottingham NG7 2UH, UK email:cj.hawkey@nottingham.ac.uk

1 Huang JQ, Lad RJ, Sridhar S, et al. H. pylori infection increases the risk of non-steroidal anti-inflammatory drug (NSAID)-induced gastroduodenal ulceration [abstract]. Gastroenterology 1998;114:A192.

2 Cullen DJ, Hawkey GM, Greenwood DC, et al. Peptic ulcer bleeding in the elderly: relative roles of Helicobacter pylori and non-steroidal antiinflammatory drugs. Gut 1997;41:459-62.

3 Labenz J, Peitz U, Köhl H, et al. Helicobacter pylori increases the risk of peptic ulcer bleeding: a case-control study. Ital f Gastroenterol Hepatol 1999;31:110-15.

4 Pilotto A, Leandro G, Di Mario F, et al. Role of Helicobacter pylori infection on upper gastrointestinal bleeding in the elderly: a case-control study. Dig Dis Sci 1997;42:586-91.

5 Wu CY, Poon SK, Chen GH, et al. Interaction between Helicobacter pylori and non-steroidal anti-inflammatory drugs in peptic ulcer bleeding [abstract]. Scand $\mathcal{F}$ Gastroenterol 1999;34:234.

6 Stack WA, Hawkey GM, Atherton JC, et al. Interaction of risk factors for peptic ulcer bleeding [abstract]. Gastroenterology 1999;116:A97.

7 Santolaria S, Lanas A, Benito R, et al. Helicobacter pylori infection is a protective factor for bleeding gastric ulcers but is not for bleeding duodenal ulcers in NSAID users [abstract]. Gastroenterology 1999;116:A231.

8 Chan FKL, Sung JJY, Chung SCS, et al. Randomised eradication of $\mathrm{H}$. pylori before non-steroidal anti-inflammatory drug therapy to prevent peppylori before non-steroidal anti-in
tic ulcers. Lancet 1997;350:975-9.

9 Hawkey CJ, Tulassy Z, Szczepanski L, et al. A randomised controlled trial of Helicobacter pylori eradication in patients taking non-steroidal antiinflammatory drugs: the HELP NSAIDs study. Lancet 1998;352:1016-21.

10 Bianchi Porro G, Parente F, Imbesi V, et al. Role of Helicobacter pylori in ulcer healing and recurrence of gastric and duodenal ulcers in long term NSAID users: response to omeprazole dual therapy. Gut 1996;39:22-6.

11 Yeomans ND, Tulassay Z, Juhasz L, et al, for the ASTRONAUT Study Group. A comparison of omeprazole and ranitidine for treating and preventing ulcers associated with non-steroidal anti-inflammatory drugs. $N$ Engl f Med 1998;338:719-26.

12 Labenz J, Tillenburg B, Peitz U, et al. Helicobacter pylori augments the $\mathrm{pH}$-increasing effect of omeprazole in patients with duodenal ulcer. Gastroenterology 1996;110:725-32. 